\title{
Mtd/Bok takes a swing: proapoptotic Mtd/Bok regulates trophoblast cell proliferation during human placental development and in preeclampsia
}

\author{
JE Ray ${ }^{1,2,3}$, J Garcia ${ }^{1}$, A Jurisicova ${ }^{1,2,3}$ and I Caniggia ${ }^{*, 1,2,3,4}$
}

We have previously reported that matador/Bcl-2 ovarian killer (Mtd/Bok), a proapoptotic member of the Bcl-2 family, regulates human trophoblast apoptosis and that its levels are elevated in severe preeclamptic pregnancy. Herein, we show that Mtd is also involved in the regulation of proliferation in normal and pathological placentae. Mtd was found in proliferating trophoblast cells during early placental development and in preeclampsia (PE). The main isoform of Mtd associated with trophoblast proliferation was Mtd-L, the full-length isoform, which preferentially localized to the nuclear compartment in proliferating cells, whereas during apoptosis it switched localization to the cytoplasm where it associated with mitochondria. Mtd expression in proliferating cells colocalized with cyclin $\mathrm{E}_{1}, \mathrm{a} \mathrm{G}_{1} / \mathrm{S}$ phase cell cycle regulator. MtdL-specific knockdown in the early first trimester villous explants and in HEK293 revealed a direct effect of Mtd-L on cyclin E1 expression and cell cycle progression. We conclude that Mtd-L functions to regulate trophoblast cell proliferation during early placentation and that the elevated levels of Mtd found in PE may contribute to increased trophoblast proliferation accompanying this devastating disorder of pregnancy.

Cell Death and Differentiation (2010) 17, 846-859; doi:10.1038/cdd.2009.167; published online 27 November 2009

In humans, early placental development is defined by an intricate balance between cellular proliferation, differentiation and death of the trophoblast lineage, and the cells forming the placenta. ${ }^{1-3}$ These cellular events are closely linked and likely regulated by many of the same molecules.

Early development takes place in a relatively hypoxic environment $(\sim 20 \mathrm{mmHg})$, whereby low oxygen acts as a key regulator of early trophoblast differentiation. ${ }^{4-6}$ In this environment, trophoblast proliferation is abundant, whereas the rate of trophoblast cell death is low. ${ }^{7}$ By $10-12$ weeks of gestation, the oxygen levels increase to $\sim 55 \mathrm{mmHg}$. This is accompanied by changes in the expression pattern of a number of gene products, ${ }^{8,9}$ and is associated with a decrease in trophoblast proliferation and an increased susceptibility to cell death. ${ }^{7,8}$ This fine-tuning of trophoblast turnover is governed by a variety of molecules expressed by the placenta, including those comprising the $\mathrm{Bcl}-2$ family. ${ }^{10,11}$

The Bcl-2 family of molecules, classically known for their involvement in the regulation of apoptosis, includes both cell death suppressors (Bcl-2, Bcl-xL, Mcl-1, A1) and cell death inducers containing either three $\mathrm{Bcl}-2$ homology $(\mathrm{BH})$ domains (Bax, Bak, and matador/Bcl-2 ovarian killer ( $\mathrm{Mtd} /$ Bok)) or inducers with only a single $(\mathrm{BH} 3)$ domain (Hrk, Bim, Bad, Bik, Noxa, and Puma). Members of this gene family act through a complex network of homo- and hetero-dimers with limited specificity. The proapoptotic multidomain members are believed to regulate apoptosis by forming channels in the outer membrane of mitochondria leading to the release of proapoptogenic factors. ${ }^{12-14}$ In recent years, it has become apparent that a number of Bcl-2 family members also regulate cell cycle progression. ${ }^{11,15-17}$ Interestingly, although antiapoptotic multidomain members slow down progression through the cell cycle, proapoptotic molecules appear to promote cell cycle progression. ${ }^{15-21}$

Matador is a proapoptotic Bcl-2 family member that is highly expressed in reproductive tissues. ${ }^{14,22}$ Its gene, Mtd, is alternatively spliced and encodes for three protein isoforms Mtd-L, Mtd-S, and Mtd- $P$, with the $L$ and $P$ isoforms predominating in the human placenta. ${ }^{14,23}$ Similar to Bax, all isoforms of Mtd contain three $\mathrm{BH}$ domains and a transmembrane domain that facilitate proapoptotic activity by mitochondrial depolarization. ${ }^{14}$ Previously, we reported that MtdL and MtdP expression is high during early placental development. ${ }^{14}$ As this period is characterized by intense trophoblast cell proliferation and little trophoblast cell death, we hypothesize that Mtd, in addition to its classical role in apoptosis, may have a function in regulating trophoblast cell proliferation. Interestingly, in vitro studies have shown that the Mtd promoter can be activated at the $G_{1} / S$ boundary by the E2F1/3 transcription factor, ${ }^{24}$ providing indirect evidence that Mtd may contribute to the regulation of cell cycle progression. However, the mode by which Mtd exerts its function in the cell cycle remains to be established.

\footnotetext{
${ }^{1}$ Samuel Lunenfeld Research Institute, Mount Sinai Hospital, Canada; ${ }^{2}$ Department of Obstetrics and Gynecology, Toronto, Ontario, Canada; ${ }^{3}$ Department of Physiology, Toronto, Ontario, Canada and ${ }^{4}$ Department of Pediatrics, University of Toronto, Toronto, Ontario, Canada

*Corresponding author: I Caniggia, Department of Obstetrics and Gynecology, Samuel Lunenfeld Research Institute, Mount Sinai Hospital, 25 Orde Street Room 6-1004, Toronto, Ontario, Canada M5 T 3H7. Tel: 416-586-4803; Fax: 416-586-5116; E-mail: caniggia @lunenfeld.ca Keywords: cyclin E1; Matador; preeclampsia; proliferation; trophoblast cell

Abbreviations: AMC, age-matched control; AS, antisense; BH, Bcl-2 homology; CT, cytotrophoblast cells; EVT, extravillous trophoblast cells; IF, immunofluorescence; LCM, laser capture microdissection; Mtd/Bok, matador/Bcl-2 ovarian killer; PCNA, proliferating cell nuclear antigen; PE, preeclampsia; S, sense; SNP, sodium nitroferricyanide (III); ST, syncytium/syncytiotrophoblast cells; TC, term control

Received 22.1.09; revised 03.8.09; accepted 21.9.09; Edited by R De Maria; published online 27.11.09
} 
Preeclampsia $(\mathrm{PE})$ is one of the leading causes of fetal and maternal death and morbidity worldwide, affecting approximately $3-5 \%$ of all pregnancies. ${ }^{25,26}$ Although the etiology of the disease remains unclear, it is accepted that the placenta has a central role in its pathogenesis, as its removal at delivery results in dissolution of the maternal symptoms. Typical hallmarks of PE include increased trophoblast cell death and a hyperproliferative immature phenotype of the trophoblast cells, ${ }^{27}$ which are in part due to a status of oxidative stress. $9,14,28,29$ These altered cellular events largely contribute to increased trophoblast turnover, hypothesized to be one of the primary culprits of the generalized maternal endothelial dysfunction responsible for the onset of clinical symptoms. Although this disease is not clinically manifested until the third trimester, it is believed to develop in the first trimester of pregnancy when the process of trophoblast differentiation begins. In addition, we have reported that MtdL and MtdP, but not MtdS, expression levels are significantly increased in preeclamptic placentae and that this is associated with increased trophoblast cell death; ${ }^{14}$ however, the relationship between $\mathrm{Mtd}$ expression and the hyperproliferative nature of preeclamptic trophoblast cells remains unexplored.

In this study, we report that Mtd is expressed in proliferative trophoblast cells during early placental development where it has a direct role in regulating cyclin $\mathrm{E} 1$ expression. Of clinical relevance, we found Mtd and cyclin E1 to be coexpressed in proliferating cytotrophoblasts from preeclamptic placentae. Our results indicate that, in addition to its role in apoptosis, Mtd may be involved in the regulation of trophoblast cell proliferation during the early stages of human placentation, and that its expression in PE may in part contribute to the hyperproliferative nature of trophoblast cells, typical of the disease.

\section{Results}

Mtd expression in proliferating trophoblast cells. To determine the pattern of Mtd protein expression in proliferating cells during early gestation, we performed duallabeled immunofluorescence (IF) analysis with antibodies against Mtd and Ki67, a common marker of proliferation ${ }^{30}$ (Figure 1). Mtd localization displayed a unique spatial and temporal pattern of expression. At 5-7 weeks of gestation, Mtd was observed primarily in the cytotrophoblast layer, displaying a prevalent nuclear localization (Figure 1a), whereas weak staining for Mtd was apparent in the syncytiotrophoblast (ST) layer (Figure 1a). By 10-13 weeks, Mtd expression became restricted primarily to the apical border of the ST, with weak nuclear and cytoplasmic expression in the trophoblast layers (Figure 1b). Ki67 expression was restricted to the nuclei of the cytotrophoblast cells (CTs) and decreased with advancing gestation (Figure 1). Localization of Mtd to Ki67-positive cells within the cytotrophoblast layer was abundant at 5-7weeks (Figure 1a), with $59 \%$ of CTs expressing both proteins (Table 1). However, Mtd expression was not exclusive to Ki67-positive cells. At 10-13 weeks, colocalization of Mtd and Ki67 was less frequent (Figure 1b) with the majority of cells (57\%) being Mtd- and Ki67-negative (Table 1).
We next investigated whether Mtd localized to Ki67-positive extravillous trophoblast cells (EVT) forming the anchoring columns. Mtd and Ki67 expression was similarly distributed throughout the column during the early first trimester (Figure 1c), with colocalization occurring in $60 \%$ of cells (Table 1). In contrast, in the late first trimester (Figure 1d), only $27 \%$ of EVT showed colocalization (Table 1). Interestingly, Mtd displayed both nuclear and cytoplasmic expression at 5-7 weeks (Figure 1c), whereas its localization became predominantly cytoplasmic by 10-13 weeks (Figure 1d).

Mtd localizes to villous trophoblast cells in the $\mathbf{G}_{\mathbf{1}}$ phase of the cell cycle. Although Ki67 can be used to indicate cell proliferation, it does not discriminate between the various stages within the cell cycle. To investigate whether Mtd was expressed in the $G_{1}$ phase of the cell cycle, we tested the colocalization of Mtd with cyclin E1, a cyclin specific to the $\mathrm{G}_{1} / \mathrm{S}$-transition phase. $^{31,32}$ Similar to Ki67, cyclin E1 expression was restricted to the nuclei of cytotrophoblast and EVT within floating villi (Figure 2a) and anchoring columns (data not shown) throughout gestation. Dual labeling of Mtd with cyclin $\mathrm{E} 1$ revealed that Mtd was present during the $\mathrm{G}_{1}$ phase of the cell cycle in the early first trimester CTs of floating and anchoring villi (Figure $2 \mathrm{a}$, data not shown), with coexpression decreasing in the late first trimester (data not shown). To confirm that Mtd expression was localized to CTs during the early first trimester, we performed coexpression studies of Mtd with the cytotrophoblast marker, E-cadherin ${ }^{33}$ (Figure 2b). It is noted that Mtd expression was not restricted to cyclin E1-positive cells, as cells that were presumably undergoing mitosis, as shown by chromosomal patterning (Figure 2c) and Ki67 (Figure 2c, bottom panel), also displayed Mtd expression in the nucleus.

Mtd expression can occur independently of cell death during early placentation. We next assessed the association of Mtd expression with the incidence of cell death during the first trimester using terminal deoxynucleotidyl transferase-mediated dUTP nick-end labeling (TUNEL), cleaved caspase-3 staining, and analysis of nuclear morphology (Figure 3a-d). Cell death assessed by these parameters was sporadic and increased with advancing gestation. The occasional cells that were positive for either TUNEL, cleaved caspase-3, or displayed fragmented nuclei in the early gestation were found in the stroma (Figure $3 b$ ), the ST (Figure 3a), the syncytial sprouts (Figure 3c, upper panel), and the distal portion of the anchoring villi (Figure 3c, lower panel). As expected, Mtd could be detected in the occasional apoptotic cell, as identified by apoptotic blebbing (Figure 3c, upper panels), and in the distal portion of the anchoring column (Figure 3c, lower panels). No sign of apoptosis was evident in the CTs (Figure 3).

Interestingly, punctuate expression of Mtd was observed in the nuclei and to a lower extent in the cytoplasm of proliferating cells (Figure $3 \mathrm{~d}$ ), whereas in apoptotic cells Mtd appeared aggregated and accumulated in the cytoplasm (Figure 3c, right panels).

Mtd-L is the predominant isoform expressed in proliferative trophoblast cells. Message expression of 


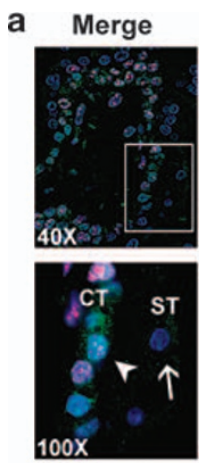

6 weeks

c
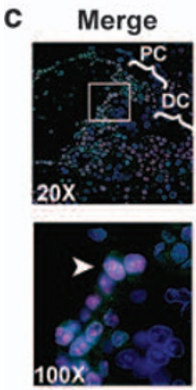

6 weeks

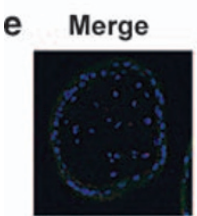

6 weeks
Mtd
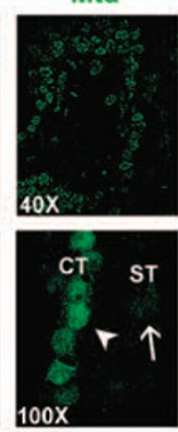

$100 x$
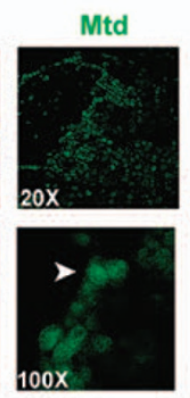

Rabbit IgG

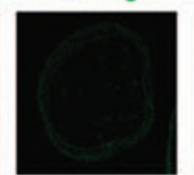

Ki67
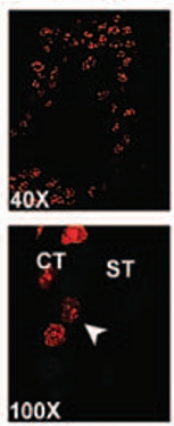

$100 x$
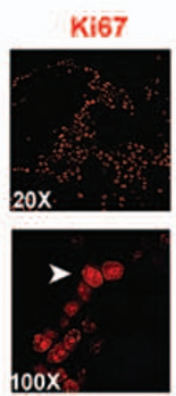

Mouse IgG

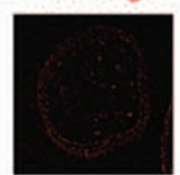

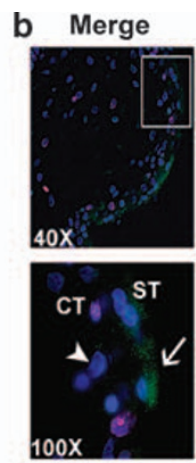

11 weeks
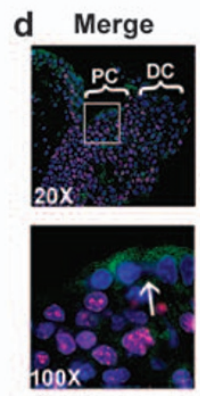

11 weeks

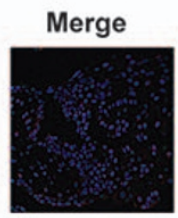

6 weeks
Mtd

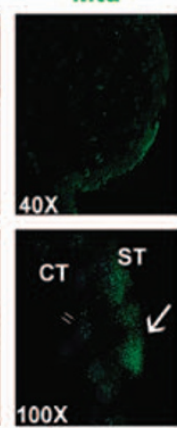

Mtd
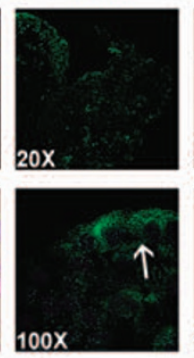

Rabbit IgG

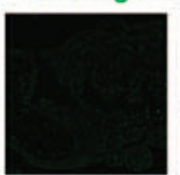

Ki67
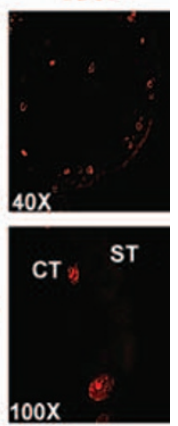

Ki87
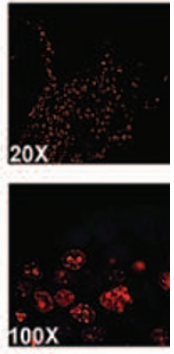

Mouse IgG

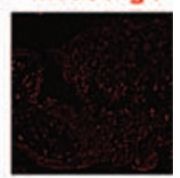

Figure 1 Mtd expression in proliferating trophoblast cells. Spatial localization of Mtd and Ki67 in placental sections from the first trimester: (a) representative early first trimester (5-8 weeks) floating villous, (b) representative late first trimester (9-12 weeks) floating villous, (c) early first trimester anchoring villi (6 weeks), and (d) late first trimester anchoring villi (11 weeks). Immunopositivity for Mtd (green), Ki67 (red), and nuclei labeled with DAPI (blue). Merged images show colocalization of Mtd, Ki67, and DAPI (overlap of red and blue: pink; overlap of green and blue: light blue; overlap of red, green, and blue: white). Lower panels show boxed regions at high magnification. (CT, cytotrophoblast; ST, syncytiotrophoblast; PC, proximal column; DC, distal column; arrow heads, nuclear positivity; arrows, cytoplasmic positivity). Panel (e) shows negative controls; first trimester floating and anchoring placental sections (6 weeks) immunostained with mouse/rabbit lgG

Table 1 Expression of Ki67 and Mtd in trophoblast cells from floating and anchoring villi during the first trimester of gestation (values are reported as percentage)

\begin{tabular}{lcccc}
\hline & $\begin{array}{c}\text { Ki67+I } \\
\text { Mtd+ (\%) }\end{array}$ & $\begin{array}{c}\text { Ki67+/ } \\
\text { Mtd-(\%) }\end{array}$ & $\begin{array}{c}\text { Ki67-I } \\
\text { Mtd+(\%) }\end{array}$ & $\begin{array}{c}\text { Ki67-I } \\
\text { Mtd- (\%) }\end{array}$ \\
\hline $\begin{array}{c}\text { Floating villi } \\
\text { 5-7 weeks }\end{array}$ & $\mathbf{5 9}$ & 13 & 18 & 10 \\
10-13 weeks & 10 & 17 & 17 & $\mathbf{5 7}$ \\
Anchoring villi & & & & \\
5-7 weeks & $\mathbf{6 0}$ & 15 & 15 & 8 \\
10-13 weeks & 27 & 14 & 19 & $\mathbf{4 0}$ \\
\hline
\end{tabular}

Mtd-L and Mtd-P, the primary isoforms of Mtd expressed by the placenta, ${ }^{14}$ was examined in proliferating versus nonproliferative trophoblast cells using laser capture microdissection (LCM), as isoform-specific antibodies toward Mtd-L and Mtd-P were unavailable.
Placental sections from 5-7 and 10-13 weeks were stained for Ki67 to identify proliferative trophoblast cell populations (data not shown). Adjacent sections were then used to isolate proliferative EVT, nonproliferative EVT, and villous trophoblast cells by LCM (Figure 4a). Both Mtd-L and Mtd-P transcripts were expressed in all three trophoblast populations examined; however, Mtd- $L$ was expressed with $C_{\mathrm{t}}$ (threshold cycle) values ranging between 24 and 29 (moderate abundance), whereas Mtd-P only exhibited $C_{\mathrm{t}}$ values ranging between 31 and 35 (very low abundance) (Figure 4b). Expression of Mtd-L in the villous trophoblast layers exhibited an increase from the early-to-late first trimester, and in the anchoring columns Mtd-L mRNA expression shifted from the proliferative EVT at 5-7 weeks to the nonproliferative EVT cell sub-population at 10-13 weeks, although this did not reach significance (Figure 4c).

Mtd isoforms are differentially localized within proliferative JEG-3 cells. Subcellular localization of specific Mtd isoforms was investigated in the human 

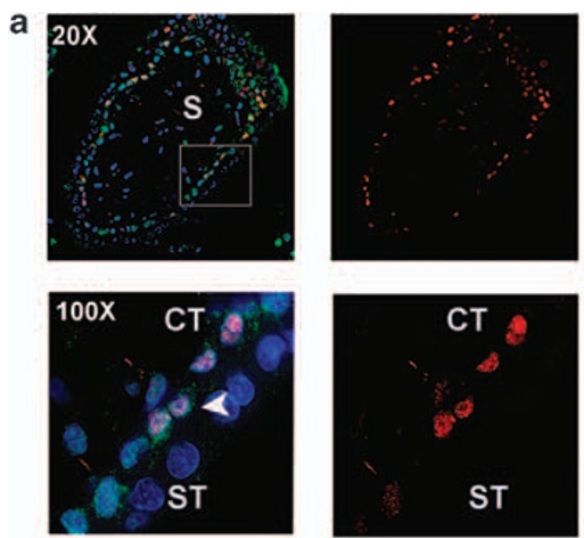

Mtd, Cyclin E1

b

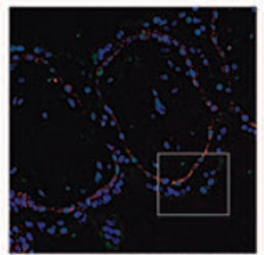

Mtd, E Cadherin

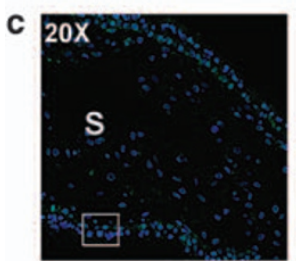

Mtd

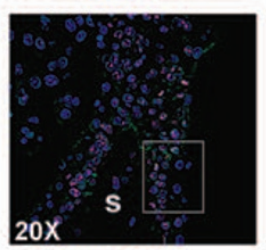

Mtd, Ki67
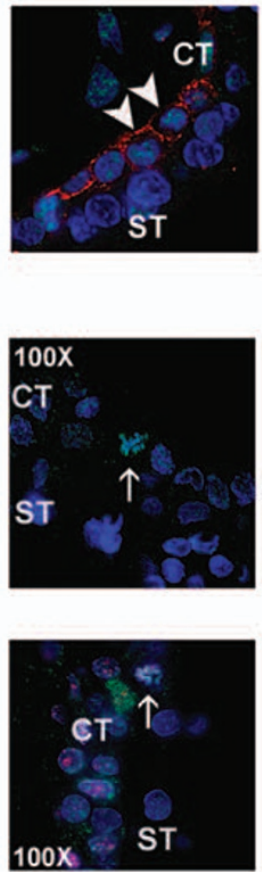
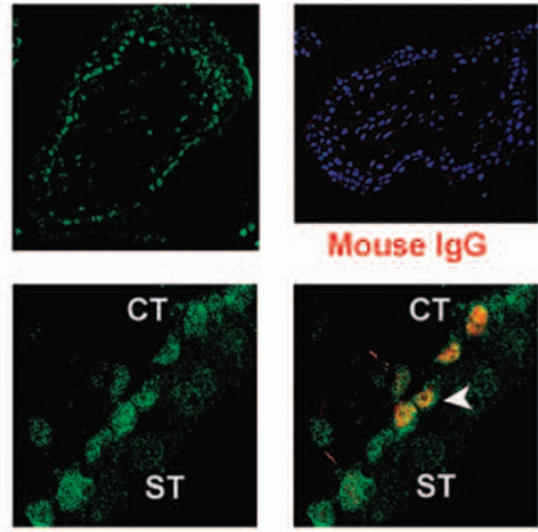

Mouse IgG
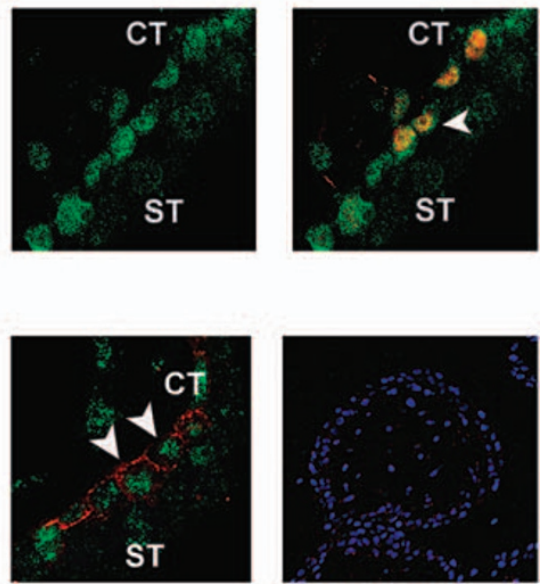

Mouse IgG
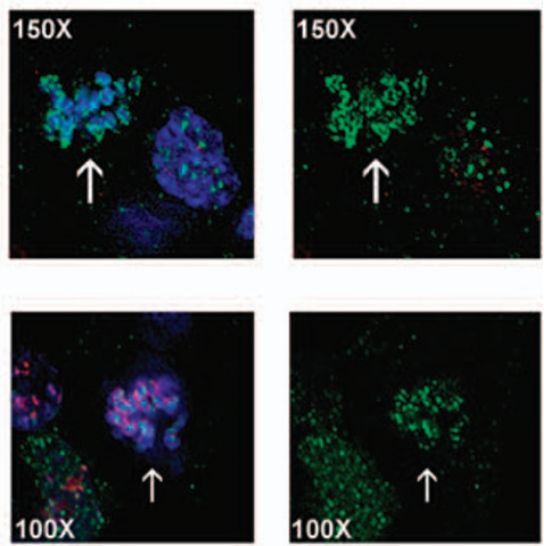

Figure 2 Association of Mtd with cyclin E1. Spatial localization of (a) Mtd and cyclin E1 in an early first trimester (6 weeks) floating villous; Mtd (green), cyclin E1 (red), nuclei detected by DAPI (blue). Merged images (left and bottom right panels) show colocalization of Mtd and cyclin E1 with or without DAPI (overlap of red, green, and blue: white; overlap of green and red: yellow). Lower panels show boxed regions at high magnification. Arrowheads, colocalization of Mtd with cyclin E1. Upper right panel, negative control. (b) Mtd expression in E-cadherin-positive trophoblast cells. Spatial localization of Mtd and E-cadherin in placental sections from a representative early first trimester (6 weeks) floating villous. Immunopositivity for Mtd (green), E-cadherin (red), and nuclei labeled with DAPI (blue). Middle and right-hand panels show boxed regions at high magnification. Arrowheads: representative cells positive for both Mtd and E-cadherin. (c) Mtd in mitotic cytotrophoblast cells; Mtd (green), Ki67 (red), and nuclei detected by DAPI (blue). Top panels: merged images show colocalization of Mtd and DAPI (overlap of green and blue: light blue). Lower panels: merged images show colocalization of Mtd, Ki67, and DAPI. Middle and right-hand panels show boxed regions at high magnification. (CT, cytotrophoblast; S, stroma; ST, syncytiotrophoblast; arrows, mitotic cells)

choriocarcinoma JEG-3 cell line under proliferative conditions. Trypan blue exclusion and 3-[4,5-dimethylthiazol-2-yl]-2,5-diphenyltetrazolium bromide (MTT) assays were used to ascertain cell viability. Cell proliferation was maximal between 24 and $48 \mathrm{~h}$ (data not shown) with a death rate below $5 \%(3.86 \pm 2.12,2.94 \pm 1.26)$. Expression of Mtd-L and Mtd-P isoforms in this cell line was confirmed by qPCR, where Mtd- $L$ was the predominant isoform expressed (Figure 5a). Subcellular fractionation showed that Mtd-L was predominantly localized to the nuclear and light membrane organelle fractions, whereas Mtd-P was found in the light membrane organelle and cytoplasmic fractions (Figure 5b).

To determine whether Mtd localizes to the mitochondria, we subjected JEG-3 cells to Mitotracker, a specific mitochondrial tracer dye, and assessed for Mtd colocalization by IF (Figure 5c-e). It was confirmed that a subset of Mtd localized to the mitochondria in healthy (Figure $5 \mathrm{~d}$ ), mitotic (Figure 5e), 
a

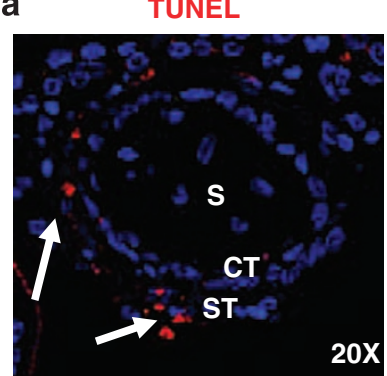

$20 x$

6week

C
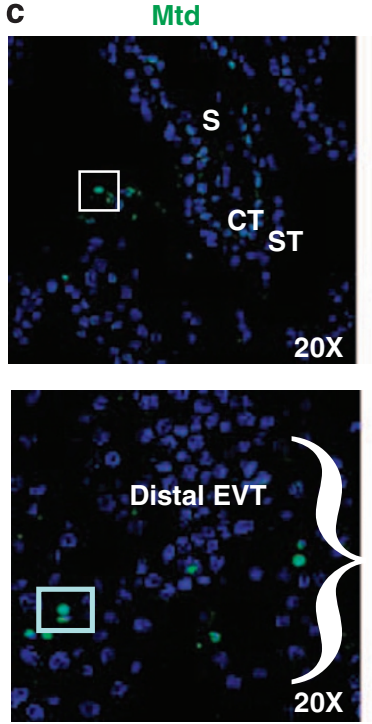

6week

d

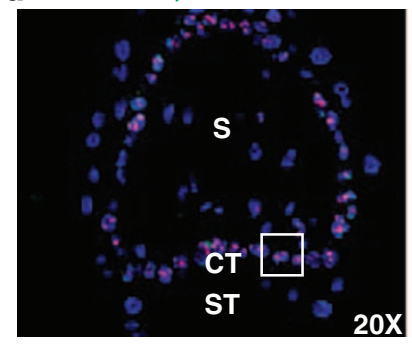

6week

$20 x$ b

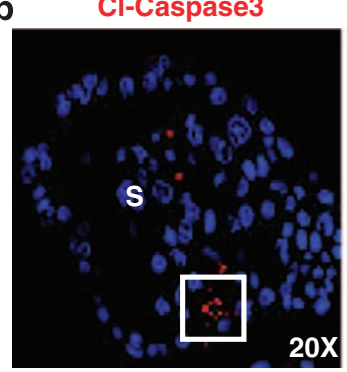

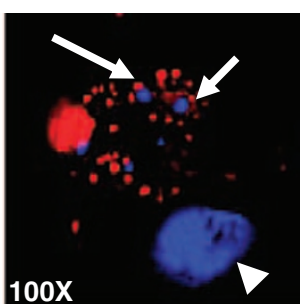

$100 x$
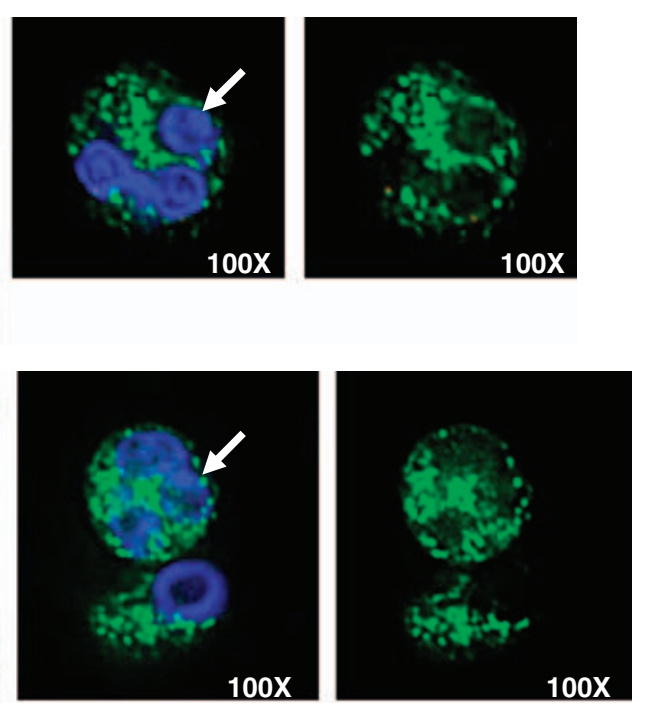

$100 x$
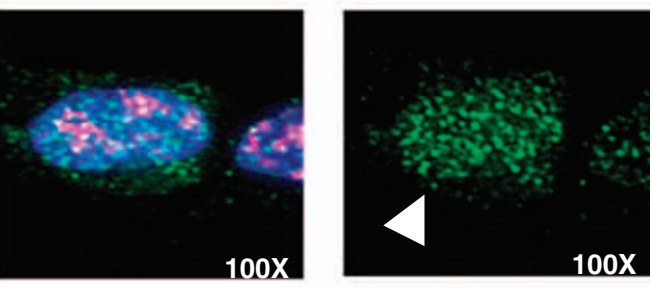

Figure 3 Apoptosis in the early first trimester placental sections. (a) TUNEL staining in a 6-week placental section. TUNEL (red) DAPI (blue). Arrow: TUNEL-positive ST cells. (b) Immunohistochemical staining for cleaved caspase-3 in a 6-week placental section. Cleaved caspase-3 (red) DAPI (blue). Right panel: higher magnification of the boxed area. Arrow: fragmented pieces of nuclei in the cleaved caspase-3-positive trophoblast cell. Arrowhead: nuclei of adjacent trophoblast cell showing no sign of apoptosis. (c) Mtd expression in apoptotic trophoblast in syncytial knots and distal EVT from the early first trimester (7-week placenta). Mtd (green), DAPI (blue). Upper panels: villi with adjacent apoptotic cell. Lower panel: distal portion of the anchoring column. Middle and right panels: high magnification of the boxed areas. Arrow: fragmented pieces of nuclei in the Mtd-positive cell. Right panel showing clumped Mtd expression in the cytoplasm of an apoptotic cell. (d) Mtd localization to cytotrophoblast cells that express the proliferative marker ki67. Mtd (green), Ki67 (red), DAPI (blue). Middle and right panels: high magnification of the boxed area. Arrowhead: punctuate expression of Mtd. (CT, cytotrophoblast; S, stroma; ST, syncytiotrophoblast; distal EVT, distal portion of extravillous trophoblast column)

and naturally occurring apoptotic cells (Figure 6a). To verify that a similar localization of Mtd is found in vivo, we examined the first trimester human placental sections dual-labeled with antibodies against Mtd and markers of various cellular organelles. No colocalization was seen between Mtd and the nuclear envelope, the endoplasmic reticulum, the Golgi apparatus, the cell membrane, or lysosomes (data not shown). However, consistent with our cell line studies, a subset of Mtd colocalized with mitochondria in the CTs (data not shown). 
a

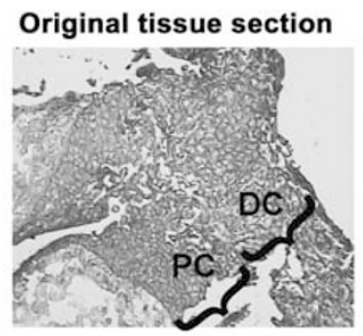

b

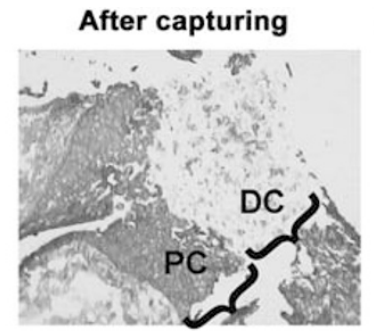

Dissected distal column

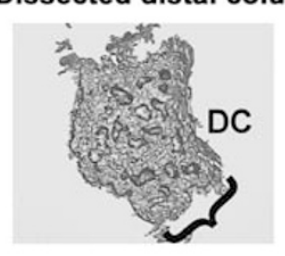

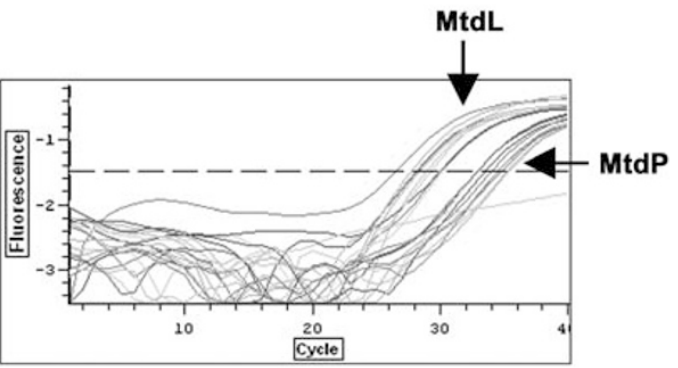

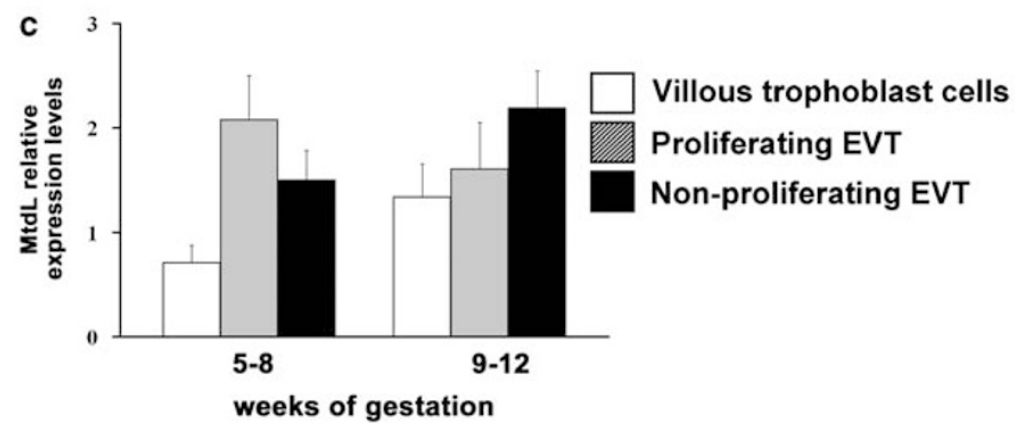

Figure 4 Mtd isoform mRNA expression in trophoblast subpopulations. (a) Laser capture microdissection of the nonproliferative, distal portion of an anchoring column (7-week placenta). (b) Expression of Mtd L and P isoforms by qPCR. Threshold cycle was taken as the point where the curve contacted the dashed line. (c) Graphical representation of Mtd-L mRNA in villous (open bars), proliferative EVT in proximal column (hatched bars), and nonproliferative EVT distal column (black bars) in the first trimester placental sections. $n=6$ for samples from 5 to 8 weeks, $n=5$ for samples from 9 to 12 weeks

SNP-induced apoptosis promotes mitochondrial localization of Mtd in JEG-3 cells. To determine whether Mtd preferentially localized to the mitochondria during apoptosis, we assessed Mtd localization in JEG-3 cells following apoptotic induction with sodium nitroferricyanide (III) (SNP), a nitric oxide donor recognized to induce apoptosis in the JEG-3 cell line. ${ }^{29}$ Cell death and viability were assessed over $48 \mathrm{~h}$ by trypan blue exclusion and MTT assays (data not shown). Two-day exposure to 2.5 and $5 \mathrm{mM}$ SNP inhibited proliferation (data not shown) and triggered cell death (2.5 mM: $23.65 \pm 4.07 \%$ and $5 \mathrm{mM}: 73.82 \pm 6.57)$. Nuclear morphology was assessed $24 \mathrm{~h}$ following treatment. Mitotic structures were readily seen in untreated conditions (Figures $5 \mathrm{c}$, e and 6a), whereas nuclear blebbing was frequently observed in both SNP-treated conditions (Figures $6 \mathrm{~b}$ and $\mathrm{c}$ ). Mtd was assessed by fluorescence immunocytochemistry in JEG-3 cells treated with mitotracker. Mtd could be seen in both the nuclear and cytoplasmic compartments of cells in the early stages of apoptosis, displaying nuclear condensation and reformation (Figure $6 \mathrm{a}-\mathrm{c}$, top right panels). With the progressive degree of apoptosis, evidenced by increased nuclear transformation and blebbing, Mtd expression became less nuclear and predominantly cytoplasmic, by which it appeared aggregated and localized to mitochondria (Figure 6b, lower panel; Figure 6c, middle panel). At late stages, Mtd could be detected in the cytoplasm but its localization to mitochondria could not be found, as the mitochondria were no longer capable of uptaking the tracer dye (Figure 6c, bottom panel).

Inhibition of Mtd-L suppresses cyclin E1 expression. To determine the functional significance of Mtd in trophoblast cell cycle during the first trimester, we evaluated the consequences of inhibiting Mtd-L on cyclin E1 expression in the first trimester human placental explants using an antisense (AS) knockdown approach. This approach has been previously used in our laboratory with a knockdown efficiency in explants of $40-60 \% .^{14}$ The inhibition of Mtd-L resulted in a 31.5 and $31.3 \%$ reduction of both cyclin E1 mRNA and protein expression, respectively (Figure 7a and $\mathrm{b}$ ). In addition, this was accompanied by a marked decrease in proliferating cell nuclear antigen (PCNA) expression, a marker of $S$ phase (Figure $7 \mathrm{~b}$, middle panel). Interestingly, knockdown of Mtd-L had no effect on cyclin E1 expression in the late first trimester of gestation (data not shown). 
a

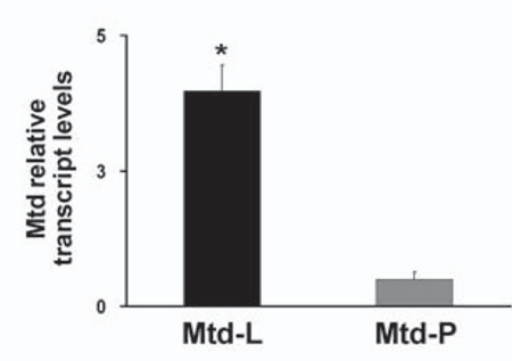

b

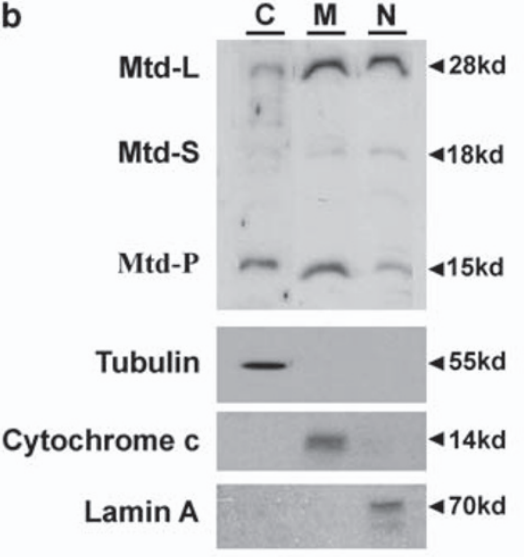

c
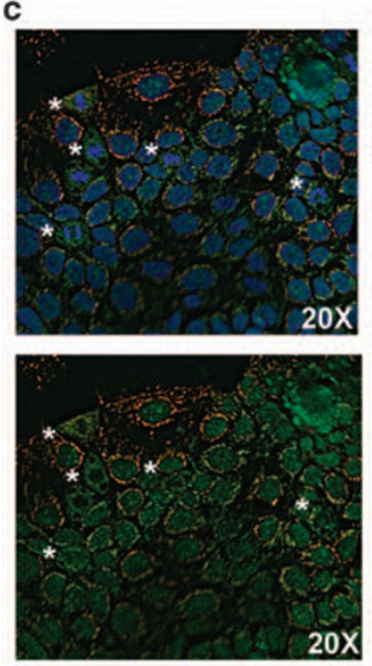

d
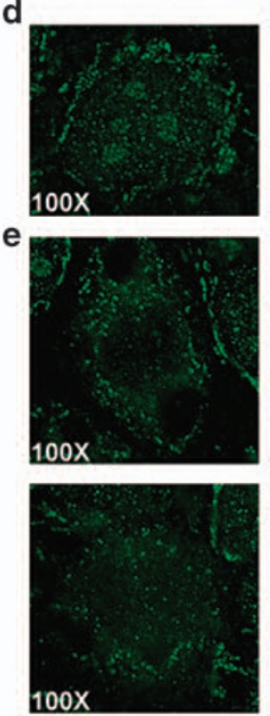
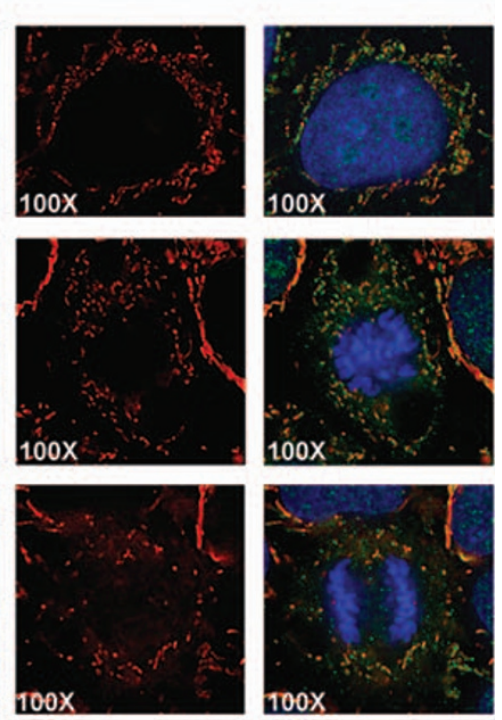

Mitotracker, Mtd, DAPI

Figure 5 Subcellular localization of Mtd isoforms in JEG-3 cells. (a) mRNA expression of Mtd-L (filled bar) and Mtd-P (hatched bar) in JEG-3 cells grown in standard conditions for $48 \mathrm{~h}$. Expression of Mtd-L, corrected against 18S, is significantly elevated compared with Mtd-P. (b). Fractionation of JEG-3 cells grown in standard conditions for $48 \mathrm{~h}$. Upper panel: western blot for Mtd. Lower panel: verification of cellular fractions: tubulin (cytoplasmic), cytochrome $c$ (mitochondrial), and lamin A (nuclear membrane). (c-e) JEG-3 cell grown in standard conditions for $24 \mathrm{~h}$ labeled with mitotracker (red) and immunostained for Mtd (green), DAPI (blue). Mitotic cells denoted by an asterisk. Colocalization of Mtd and mitochondria: yellow (overlap of red and green). (d) JEG-3 cell at high magnification displaying healthy nuclear morphology, (e) high magnification of JEG-3 cells in mitosis. ${ }^{*} P<0.01$, Mann-Whitney U-test

We have previously reported that overexpression of MtdL and MtdP results in cellular apoptosis. ${ }^{14}$ To establish a role for Mtd in cellular proliferation, we generated a doxycyclineinducible MtdL expression system in HEK293 cells, which allows for controlled MtdL expression (Figure 7c). Induction of MtdL using $0.25 \mu \mathrm{g} / \mathrm{ml}$ doxycycline over $36 \mathrm{~h}$ did not affect the rate of cell death as determined by trypan blue exclusion (data not shown), and it was associated with a $32 \%$ increase in cyclin E1 protein expression (Figure 7c). This was accompanied by an increase in 5-Bromo-2'-deoxy-uridine (BrdU) incorporation by these cells (Figure 7d). Conversely, an siRNA-mediated reduction of MtdL (75\%) in HEK293 cells led to a decrease in both cyclin E1 protein expression (41\%) and BrdU incorporation (Figure $7 f$ and $g$ ).

Mtd expression in proliferating trophoblast cells in PE. We have previously reported that Mtd expression is elevated in placentae from severe preeclamptic pregnancies and that this is associated with increased trophoblast cell death, characteristic of this disease. To determine whether the increase in Mtd was also associated with the hyperproliferative nature of the trophoblast cells, we tested whether Mtd was expressed in Ki67- and cyclin E1-positive cells in placental samples from PE. As anticipated, PE displayed greater levels of Ki67 (\% of Ki67-positive trophoblast cells, PE: $8.6 \%$ versus age-matched control (AMC): $3.7 \%$ and term control (TC): $1.7 \% ; P<0.05)$, cyclin E1 ( $\%$ of cyclin E1-positive trophoblast cells, PE: $13 \%$ versus AMC: $6.3 \%$ and TC: $2.5 \% ; P<0.05$ ), and Mtd compared with agematched and TCs (Figure 8a-d). Furthermore, dual labeling of Mtd with Ki67 and cyclin E1 was evident to a greater extent in preeclamptic samples compared with either age-matched (Figure $8 \mathrm{a}$ and $\mathrm{c}$ ) or TCs (data not shown). Interestingly, nuclear expression of Mtd in preeclamptic samples was 

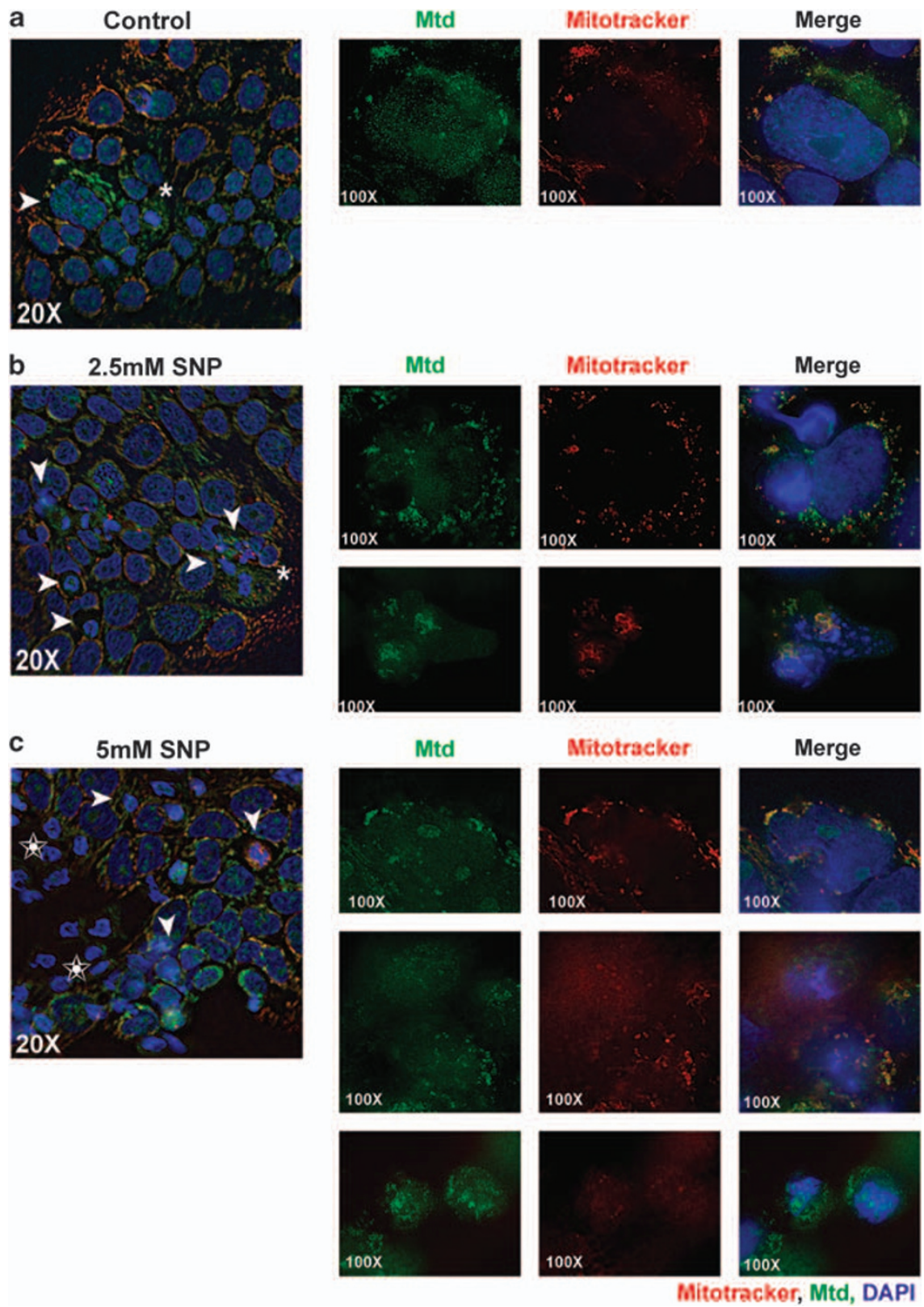

Figure 6 Localization of Mtd to mitochondria in apoptotic JEG-3 cells. JEG-3 cells treated with or without SNP for $24 \mathrm{~h}$. (a) untreated JEG-3 cells. (b) JEG-3 cells treated with $2.5 \mathrm{mM}$ SNP. (c) JEG-3 cells treated with $5 \mathrm{mM}$ SNP. Cells were labeled with mitotracker and immunostained for Mtd. Mitotracker (red) Mtd (green), DAPI (blue). Colocalization of Mtd and mitochondria: yellow (overlap of red and green). Arrowhead denotes apoptotic cells; Star denotes area of apoptotic cells; Asterisk denotes mitotic cells. $\times 100$ images show apoptotic cells representative from each treatment group. (a) Occasional apoptotic cell in untreated group displaying early stages of apoptosis and colocalization of Mtd with mitotracker. (b) Cells treated with $2.5 \mathrm{mM}$ SNP showing the occasional mitotic structure and increased levels of cell death with early and late stages of apoptosis. (c) Numerous apoptotic cells displaying a range from early (condensed, blebbing nuclei, top right panel) to blebbing and fragmented nuclei (middle panel) and apoptotic bodies (lower panel)

predominant in Ki67- or cyclin E1-positive cells, whereas in the syncytial knots the expression was mainly cytoplasmic. In contrast, comparable levels of Mtd were seen in both the nuclear and cytoplasmic regions of age-matched (Figure 8a and $\mathrm{c}$, bottom panels) and TC sections (data not shown). Coexpression of Mtd with E-cadherin confirmed that CTs were the predominant sites of nuclear Mtd expression in preeclamptic placentae (Figure 8e).

\section{Discussion}

In recent years, it has become apparent that members of the Bcl-2 family are involved in regulating cell fate in a variety of systems. ${ }^{15-17}$ Data presented herein show that Mtd, a proapoptotic molecule of the $\mathrm{Bcl}-2$ family, is involved in the regulation of both proliferation and cell death in the human placenta in physiological and pathological conditions. In particular, we show that (1) Mtd is expressed in proliferating 

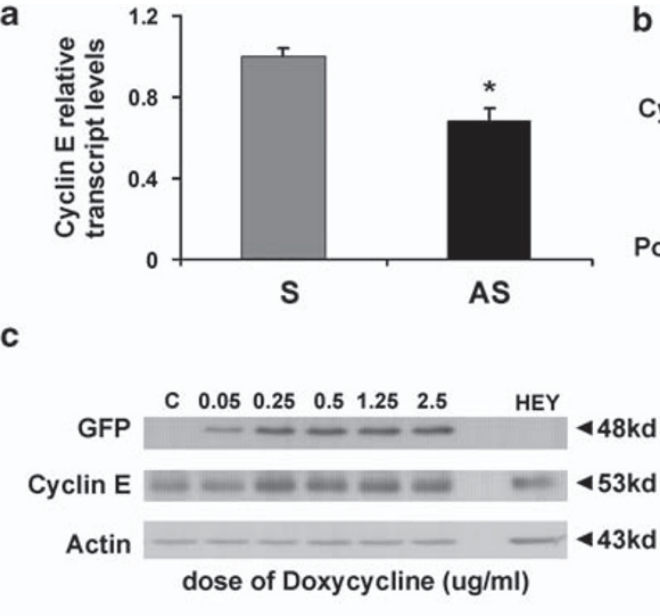

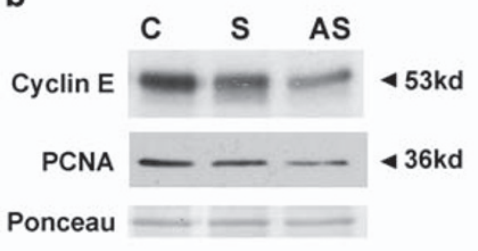

d Control
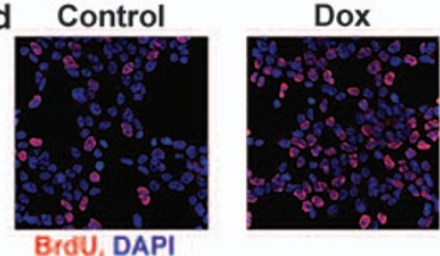

f
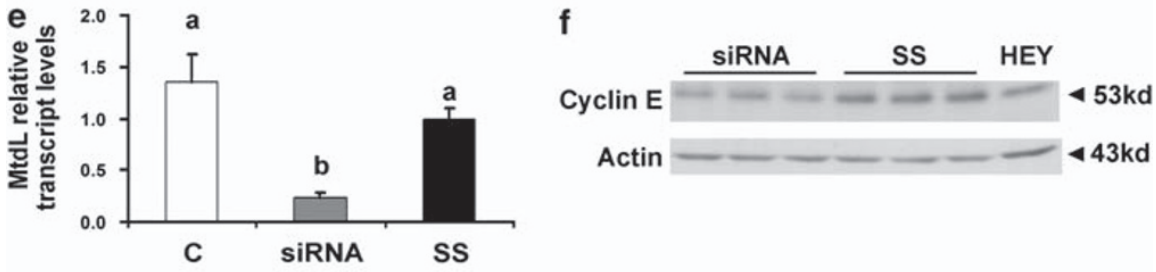

g SS SiRNA

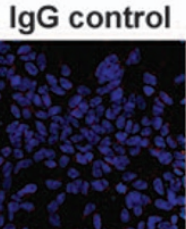

\section{BrdU, DAPI}

Figure 7 The effect of Mtd-L interference on cyclin E1. (a, b) Expression of cyclin E1 (mRNA: $n=4$; protein: $n=4$ ) in the early first trimester explants treated with Mtd-L sense (S, nonsilencing) oligonucleotides or Mtd-L antisense oligonucleotides (AS, silencing) or untreated (C) in $3 \% \mathrm{O}_{2}$ for $72 \mathrm{~h}$. (a) Relative transcript levels of cyclin E1 as detected by qPCR and corrected against $18 \mathrm{~S}$. ${ }^{*} P<0.05$, Mann-Whitney U-test. (b) Expression levels of cyclin E1 protein (upper panel) and PCNA protein (middle panel). Confirmation of equal loading by Ponceau detection of total proteins (lower bands). (c) Induction of GFP-hMtdL in Flp-In T-Rex-293 cell line with increasing concentrations of doxycycline $(0.05,0.25,0.5,1.25$, and $2.5 \mu \mathrm{g} / \mathrm{ml})$ over a $36 \mathrm{~h}$ period. HEY ovarian cancer cell lysate was included as a negative control for GFP and positive control for cyclin E1. Top panel: level of hMtdL expression detected by GFP. Middle panel: expression of cyclin E1 and bottom panel, control actin. (d) BrdU incorporation in hMtdL Flp-In T-Rex-293 cell line following treatment with or without $0.25 \mu \mathrm{g} / \mathrm{ml}$ doxycycline over a $36 \mathrm{~h}$ period. $(\mathbf{e}-\mathbf{g})$ knockdown of MtdL in HEK293 cells using siRNA strategy. (e) Fold changes in MtdL transcript levels relative to scrambled sequence (SS) as detected by qPCR. Statistical significance was assessed by Kruskal-Wallis test followed by Mann-Whitney U-tests; a, b, c: $P<0.05$. (f) Expression levels of cyclin E1 protein and actin. (g) BrdU incorporation in HEK293 following knockdown of MtdL using $30 \mu \mathrm{M}$ siRNA for $48 \mathrm{~h}$. Negative control; immunostaining with mouse IgG in HEK cells subjected to BrdU

trophoblast cells during early placental development and in severe early onset $P E$, where it acts on the $G_{1}$ phase of the cell cycle by directly regulating the expression of cyclin $\mathrm{E} 1,(2)$ $M t d-L$ is the isoform responsible for this effect on cell proliferation, and (3) Mtd localizes to the nuclear compartment in proliferating cells, whereas during apoptosis it switches localization to the cytoplasm where it interacts with mitochondria.

Both the antiapoptotic and proapoptotic members of the $\mathrm{Bcl}-2$ family have been shown to participate directly in cell cycle regulation independent of their apoptotic function. ${ }^{15-17}$ The antiapoptotic members, Bcl-2, BCL-xL, BCL-w, and Mcl-1, have been shown to have inhibitory effects on passage through the cell cycle, whereas the proapoptotic member, Bax, promotes cell proliferation by conferring cell cycle advancement. ${ }^{17,18,21}$ Although the Mtd promoter has been found to be activated at the $G_{1} / S$ boundary in vitro, ${ }^{24}$ we present for the first time direct evidence that the Mtd protein is expressed in cycling cells in vivo. In the human placenta, the expression of Bax associates predominantly with the apoptotic index, ${ }^{34}$ suggesting that Bax may not be involved in trophoblast cell cycle regulation. This underscores a unique function for Mtd as a proapoptotic regulator of cell cycle progression in the human placenta.

Our study found that nuclear expression of Mtd associated closely with the proliferative index throughout human placental development. In the early first trimester, Mtd localized primarily to the nucleus in Ki67- and cyclin E1positive CTs that displayed healthy nuclear morphology with no sign of apoptosis, whereas past the ninth week of gestation, as the percentage of proliferative cells decreased, expression of Mtd switched to the apical border of the ST layer. The effect of Mtd on cyclin E1 was also associated with this temporal regulation, occurring only in the early first 

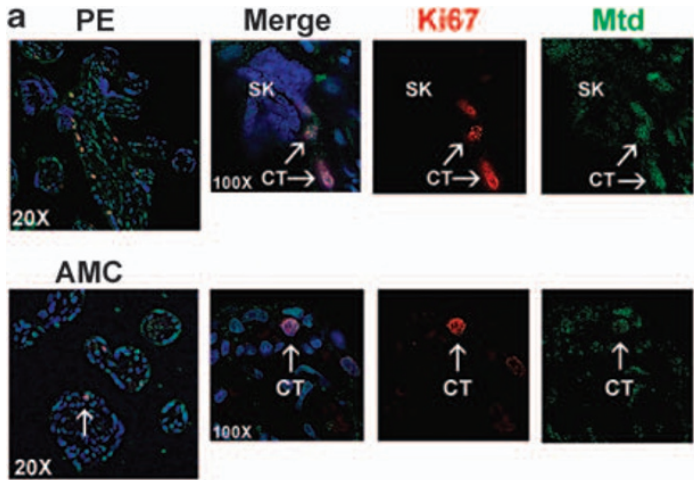

b

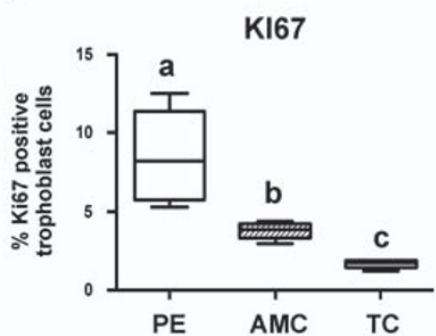

C PE
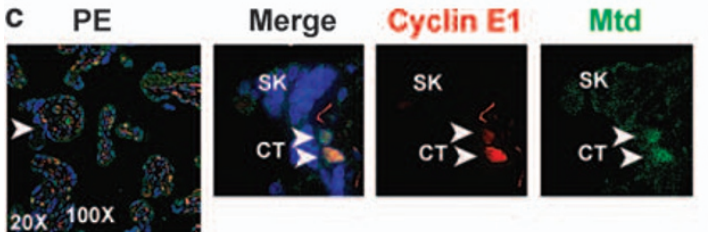

AMC
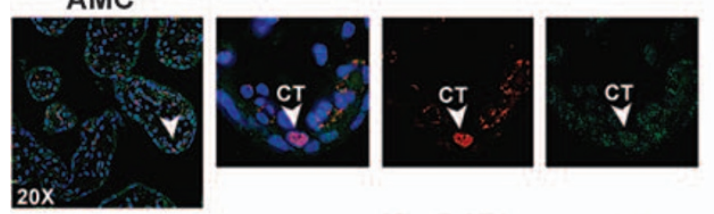

d

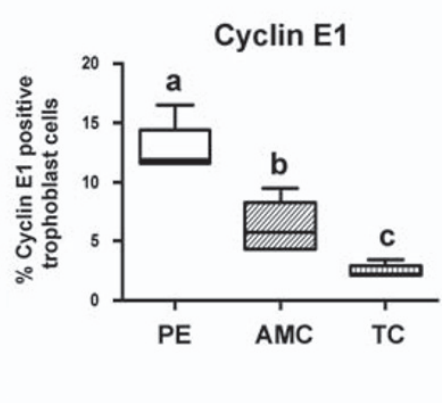

e PE
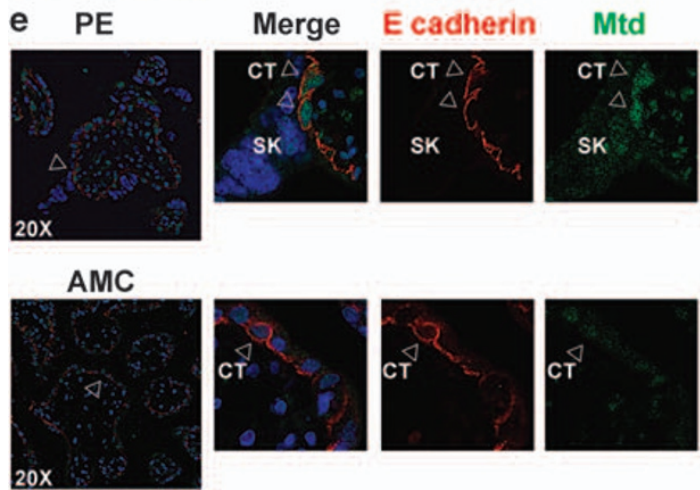

Figure 8 Localization of Mtd to proliferative cells in preeclamptic placentae. (a) Spatial localization of Mtd-L and Ki67 in placental tissue from preeclamptic (PE) and agematched control (AMC) placentae. Mtd (green), Ki67 (red), and DAPI (blue). Top panels: floating villi from a preeclamptic placenta at 29 weeks of gestation, bottom panels: floating villi from an age-matched control placenta (34 weeks). Left-hand panels: $\times 20$ magnification; middle and right panels: $\times 100$ magnification. Merged panels show colocalization (yellow/white) of Mtd with Ki67 with nuclei detected by DAPI staining. CT, cytotrophoblast cells; SK, syncytial knots; arrows, colocalization of Mtd and Ki67 in the nuclear compartment of cells in the trophoblast layer. (b) Quantification of the percentage of trophoblast cells positive for Ki67 in PE $(n=5)$, AMC $(n=5)$, and term controls (TC) $(n=5)$. Statistical significance was assessed by Kruskal-Wallis test followed by Mann-Whitney U-tests; a, b, c: $P<0.05$. (c) Spatial localization of Mtd and cyclin E1 in placental tissue from preeclamptic and age-matched control placentae. Mtd (green), cyclin E1 (red), and DAPI (blue). Top panels: floating villi from a preeclamptic placenta at 26 weeks of gestation, bottom panels: floating villi from an age-matched control placenta (32 weeks). Left-hand panels: $\times 20$ magnification; middle and right panels: $\times 100$ magnification. Merged panels show colocalization (yellow/white) of Mtd with cyclin E1 with nuclei detected by DAPI staining. CT, cytotrophoblast cells; SK, syncytial knots; arrowhead, colocalization of Mtd and cyclin E1 in the nuclear compartment of cells in the trophoblast layer. (d) Quantification of percentage of trophoblast cells positive for cyclin E1 in PE $(n=5)$, AMC $(n=5)$, and term controls (TC) $(n=5)$. Statistical significance was assessed by Kruskal-Wallis test followed by Mann-Whitney U-tests; a, b, c: $P<0.05$. (e) Spatial localization of Mtd and the cytotrophoblast marker E-cadherin in placental tissue from preeclamptic and age-matched control placentae. Mtd (green), E-cadherin (red), and DAPI (blue). Top panels: floating villi from a preeclamptic placenta (32 weeks), bottom panels: floating villi from an age-matched control placenta (32 weeks). Left-hand panels: $\times 20$ magnification; middle and right panels: $\times 100$ magnification. Merged panels show coexpression of Mtd with E-cadherin (CT, cytotrophoblast cells; SK, syncytial knots; open arrowhead, cytotrophblast cell coexpressing Mtd and E-cadherin)

trimester explants. Nonetheless, the apoptotic role of Mtd has been shown to be maintained past the first trimester. ${ }^{14}$ Taken together, these data suggest that a change in Mtd function may take place late in the first trimester, away from a cell cycle regulatory role, and that this is accompanied by a change in subcellular localization. This switch in localization and function is likely regulated by the increase in oxygen concentration experienced by the placenta at this time. This is supported by previous studies that have found hypoxia response elements in the promoter region of $M t d,{ }^{14,35}$ 
and have shown Mtd to be decreased in higher oxygen conditions. ${ }^{14,35}$

As the inhibition of Mtd leads to a decrease in cyclin E1, we hypothesize that Mtd may function during the $\mathrm{G}_{1} / \mathrm{S}$ transition. In accordance with our observations, a recent study performed with a mouse fibroblast cell line showed expression of the Mtd transcript to be increased at the mid-to-late $G_{1}$ phase, following the overexpression of $G_{1} / S$-phase transition factors. ${ }^{24}$ Moreover, the decrease in PCNA and BrdU incorporation following Mtd knockdown and increased BrdU incorporation following doxycycline-induced Mtd overexpression indicates that the effect on cyclin $\mathrm{E} 1$ expression results in altered cell cycle progression to the $S$ phase. In addition, we also observed Mtd expression in mitotic cells and in Ki67negative cells. It is possible that, similar to Mcl-1, which functions at both the $\mathrm{G}_{0} / \mathrm{G}_{1}$ and $\mathrm{G}_{2} / \mathrm{M}$ borders, ${ }^{19,20}$ Mtd may have different roles at distinct phases of the cell cycle. ${ }^{19,20}$

Our data also suggest that the role of Mtd in cell fate is likely dependent on conformation and location of the protein. This mode of regulation has previously been shown for $\mathrm{Bax}, \mathrm{a} \mathrm{Bcl}-2$ family member structurally similar to Mtd. ${ }^{22,36}$ Although Bax is cytoplasmic and monomeric in healthy cells, its apoptotic function depends on its oligomerization and translocation to the mitochondria. ${ }^{37-40}$ Similarly, we observed a diffuse, punctuate expression pattern of Mtd in proliferative trophoblast cells, whereas cells undergoing apoptosis displayed a clumped or aggregated pattern of Mtd expression, consistent with oligomerization. Furthermore, Mtd has previously been reported to form oligomers under apoptotic stimuli in HEK293 cells. $^{35}$

In addition, Mtd expression was both cytoplasmic and nuclear in healthy proliferating cells, whereas its expression became cytoplasmic and localized to the mitochondria in cells undergoing apoptosis. Mtd has been shown to interact with the exportin Crm $1,{ }^{41}$ supporting the idea that Mtd can travel between the nuclear and the cytoplasmic compartments to exert its cellular function. It is therefore plausible that in trophoblast cells, low levels of Mtd remain monomeric and locate to the nucleus, where their function is linked to cell cycle regulation, whereas cytoplasmic accumulation of Mtd promotes oligomerization, localization to the mitochondria, and a functional switch toward its apoptotic role.

Interestingly, Mtd was also observed in a subset of mitochondria in healthy JEG-3 cells. This may be explained by previous work showing that in healthy MCF-7 cells, Mtd loosely associates with the mitochondria and that on apoptotic stimulation, Mtd becomes tightly integrated into the mitochondrial membrane. ${ }^{35}$

Mtd is expressed as three isoforms in total human placental lysate, the principal isoforms being Mtd-L and Mtd-P. ${ }^{14}$ We postulate that the Mtd- $L$ isoform has a dual role in both cell proliferation and death, whereas Mtd-P may primarily have a 'killing' role. This is supported by our fractionation studies that revealed Mtd-L to be the predominant isoform expressed in the nuclear compartment of proliferating JEG-3 cells, where it would have direct access to interaction with cell cycleregulating molecules, and by our knockdown and overexpression experiments where Mtd- $L$ was seen to have a direct effect on cyclin E1 expression. In contrast, both Mtd-L and Mtd-P were located in the mitochondrial fraction of JEG-3
Physiologic Low Oxygen

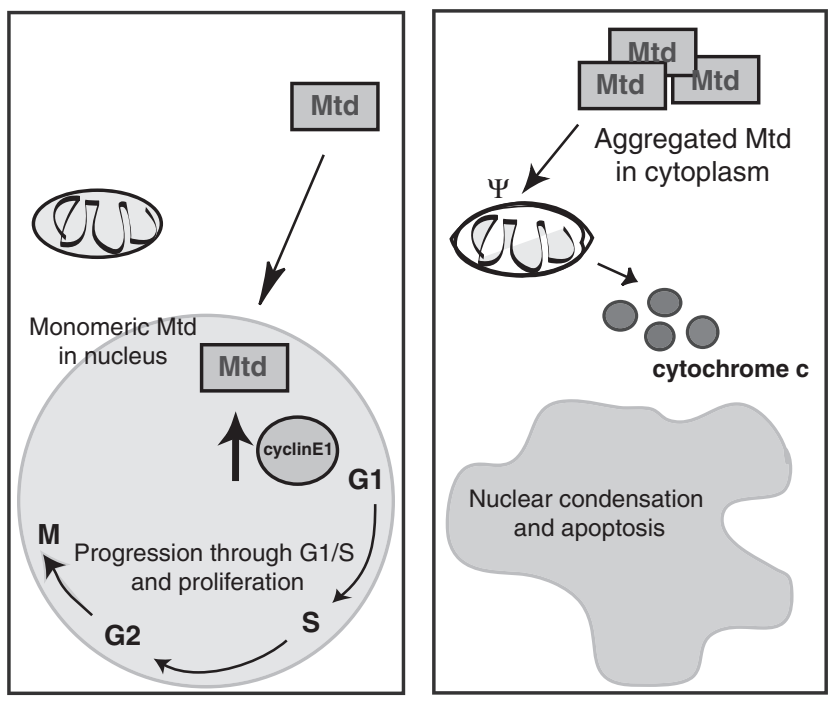

Figure 9 Putative model of Mtd function in the trophoblast. Under low oxygen conditions, as seen in early placental development, Mtd remains monomeric and localizes to the nucleus of cytotrophoblast cells where it promotes proliferation by aiding in the $G_{1}$ to $S$ transition. Under conditions of oxidative stress, Mtd accumulates in the cytoplasm where it interacts with the mitochondria. Mtd pore formation in the mitochondria leads to release of apoptogenic molecules into the cytoplasm, thus activating the apoptotic cascade and cell death

cells and their overexpression has been shown to result in apoptosis. $^{14}$

We have previously shown that both the Mtd-L and Mtd-P isoforms are increased in preeclamptic placental tissue compared with AMC and TCs. ${ }^{14} \mathrm{PE}$ is a placental disorder, characterized by hyperproliferation of the trophoblast cells and accompanied by excessive apoptosis and syncytial shedding. We therefore hypothesized that Mtd may in part contribute to the increase of both cellular apoptosis and cell proliferation in this pathology. We have previously shown that in PE, Mtd is associated with mitochondrial depolarization and an increase in apoptosis, ${ }^{14}$ and in this study we show colocalization of Mtd to the Ki67- and cyclin E1-positive CTs in $\mathrm{PE}$, suggesting that the abundance of Mtd may also contribute to the hyperproliferative phenotype. This is further supported by our data showing that in $\mathrm{PE}$, the hyperproliferative phenotype of the trophoblast cells is associated with increased Ki67 and cyclin E1 expression levels. It is likely that Mtd works in concert with a variety of cell cycle-regulating molecules, including the Cip/Kip family of inhibitors and the Notch proteins, which have also been found to be overexpessed in PE as reviewed by Heazell and Crocker. ${ }^{10}$

On the basis of a putative working model (Figure 9), we postulate that during early placental development, Mtd- $L$ is upregulated by the low oxygen environment, and that it localizes to the nucleus of the CTs where it promotes proliferation by aiding in the $G_{1}$ to $S$ transition. Introduction of apoptotic stimuli may lead to cytoplasmic accumulation of Mtd, and result in Mtd oligomerization and interaction with the mitochondria, thus producing a proapoptotic response. In conclusion, Mtd appears to have an important role in the 
proliferative and apoptotic pathways that mediate trophoblast cell fate. Improper regulation of Mtd, as seen in PE, may have a role in altering the homeostasis of trophoblast layers, contributing to the increased rate of proliferation and apoptosis associated with this pathology.

\section{Materials and Methods}

Placental tissue collection. The first trimester human placental tissues (5-13 weeks of gestation, $n=35$ ) were obtained immediately following the elective termination of pregnancies by dilatation and curettage, or suction evacuation. Gestational age was determined by the date of the last menstrual period and first trimester ultrasound measurement of crown-rump-length. Preeclamptic group $(\mathrm{PE}, n=8)$ was selected on the basis of ACOG clinical and pathological criteria. ${ }^{42}$ Controls were selected as AMC $(n=9)$ or TC $(n=8)$ healthy pregnancies with normally grown fetuses that did not have signs of placental dysfunction. Birth weight, gestational age, laboratory values, and clinical observations relevant to the health of the mother were taken from the clinical records. The PE patients' group was characterized by an average gestational age of 32 weeks; average blood pressure 168/105 (systolic/diastolic), and the presence of proteinuria (average $3 \mathrm{~g} /$ day). One out of eight PE pregnancies was associated with intrauterine growth restriction. The AMC and TC patients were delivered at an average of 31.5 and 38 weeks, respectively, and exhibited normal blood pressure (116/73) with no sign of proteinurea. Patients with diabetes, infections, and kidney disease were excluded. Placenta samples with calcification, necrosis, and visually ischemic areas were also excluded from the collection. In both the normal and pathological groups, babies were delivered by cesarean section. Normal preterm deliveries were attributed to multiple pregnancies without discordancy, preterm labor owing to an incompetent cervix, and the premature preterm rupture of a membrane. Term controls were included as pregnancies delivered preterm are by definition abnormal and may be associated with placental abnormality. ${ }^{43,44}$ Informed consent was obtained from each individual patient, and tissue collections were approved by the Mount Sinai Hospital's Review Committee on the Use of Human Subjects and carried out in accordance with the participating institutions' ethics guidelines. Tissues were snapfrozen or prepared in PFA and processed for histochemical analysis.

Samples for LCM were prepared immediately after collection. Briefly, fresh tissue was washed in PBS, saturated in cryoprotect solution $(66 \%$ OCT in 33\% sucrose solution), and embedded in OCT (optimal cutting temperature) compound (TissueTek, Sakura Finetek, Torrance, CA, USA) snap-frozen and kept at $-80^{\circ} \mathrm{C}$ until further use. Cryosections, ( $7 \mu \mathrm{m}$ thick) were cut using RNAse-free blades and mounted on uncoated, uncharged slides (Superfrost, Fisher Scientific, Ottawa, ON, Canada).

First trimester villous explant culture and Mtd antisense knockdown. Chorionic villous explant culture and Mtd knockdown was performed as previously described ${ }^{14,45}$ using phosphorothiolated (all positions) sense (S) and AS oligonucleotides designed against the Mtd-L transcript NM_032515 (S-L: 50-CATGGAGGTGCTGCGG-30, AS-L: 50-CCGCAGCACCTCCATG-30). Villous explants were incubated at $3 \% \mathrm{O}_{2}$ in the presence or absence of $S$ or $A S$ oligos $(10 \mathrm{mM})$ or DMEM/F12 alone for $72 \mathrm{~h}$. Explants from eight different first trimester placentae (6-8 weeks) run in triplicate were used for the antisense knockdown experiments.

Laser capture microdissection. Before lasercapture microdissection, immunostaining for Ki67 was performed initially to identify areas of proliferation. Briefly, frozen sections were fixed in $4 \% \mathrm{PFA}$, treated with $0.3 \% \mathrm{H}_{2} \mathrm{O}_{2}$, blocked with $5 \%$ horse serum, and incubated with anti-Ki67 antibody (dilution $1: 100$ ) for $1 \mathrm{~h}$ at room temp. LCM was performed on adjacent cryosections, using the Arcturus Pixcell II system (Arcturus Engineering, Mountain View, CA, USA) according to the manufacturer's protocol. Briefly, sections were overlaid with a thermoplastic membrane (CapSure LCM Caps, Arcturus), and cells were captured by focal melting of the membrane through laser activation. Three individual caps were used for each placental sample, specifically isolating the villous trophoblast layer comprising both CTs and STs, the proliferative (Ki67-positive) region of the proximal column, or the nonproliferative (Ki67-negative) cells of the distal anchoring columns. Approximately $2000-5000$ cells were captured onto each cap.

RNA analysis. RNA was extracted from frozen placental tissue using an Rneasy Mini Kit (Qiagen, Valencia, CA, USA) and from LCM samples using the
PicoPureRNA Isolation Kit (Arcturus). All samples were treated with DNase I, reverse transcribed using random hexamers (Applied Biosystems, Foster City, CA, USA), and amplified by 20 cycles of PCR $\left(5 \mathrm{~min}\right.$ at $95^{\circ} \mathrm{C}$, cycle: $30 \mathrm{~s}$ at $95^{\circ} \mathrm{C}, 30 \mathrm{~s}$ at $55^{\circ} \mathrm{C}$, and $1.5 \mathrm{~min}$ at $72^{\circ} \mathrm{C}$ ). Analysis was carried out using the DNA Engine Opticon 2 System (MJ Research, Waltham, MA, USA) as previously described. ${ }^{14}$ Mtd was quantified using the SYBR Green I dye DyNamo HS kit (MJ Research) based on the manufacturer's protocol using isoform-specific primers (Mtd-L: Forward $5^{\prime}$-GCCT GGCTGAGGTGTGC-3', Mtd-P: Forward 5'-GCGGGAGAGGCGATGA, Reverse (both $L$ and P) $5^{\prime}$-TGCAGAGAAGATGTGGCCA-3'). TaqMan Universal MasterMix and specific Taqman primers and probe for cyclin E1 and 18S were purchased from $\mathrm{ABI}$ as Assays-on-Demand for human genes. Data were normalized against expression of $18 \mathrm{~S}$ ribosomal RNA using the $2 \Delta \Delta C_{\mathrm{t}}$ formula as previously described. ${ }^{14}$

Antibodies. A rabbit polyclonal antibody generated against peptide mapping within an internal region of Mtd (137-151) of human origin (NM_032515) was raised in our laboratory, and preimmune serum was used as control (WB 1:500). Antibodies were purchased from Cell Signaling Technology, Beverly, MA, USA: $\mathrm{Mtd} / \mathrm{Bok}(4521)$ rabbit polyclonal (IF 1:50), and Cytochrome $c$ (136F3) rabbit monoclonal (WB 1:200); Santa Cruz Biotechnology, Santa Cruz, CA, USA: Mtd/ Bok (H151) rabbit polyclonal (IF 1:400), Cyclin E1 (HE12) sc-247 mouse monoclonal (IF 1:400, WB 1:1500), Lamin A (C20) sc-6214 goat polyclonal WB $1: 200)$, GFP (B-2) sc-9996 mouse monoclonal (WB 1:500), and Actin (I-19) sc1616 goat polyclonal (WB 1:1500); Vector Laboratories, Burlingame, CA, USA: Ki67 (clone MM1) mouse monoclonal (IF 1: 100); and Sigma-Aldrich, St. Louis, MO, USA: $\alpha$-Tubulin (clone DM 1A) mouse monoclonal (WB 1:2000); DakoCytomation, Denmark A/S: PCNA (clone PC10) mouse monoclonal (WB 1:500) Abcam, Cambridge, MA, USA: E-Cadherin (HECD-1) mouse monoclonal (IF 1:200) and Roche, Mannheim, Germany: BrdU mouse monoclonal (IF 1:100). Normal rabbit and mouse IgG (sc-2027 and sc-2025, respectively) were purchased from Santa Cruz Biotechnology and used as negative control. Secondary antibodies; horseradish peroxidase-conjugated or biotinylated anti-rabbit/mouse/goat IgG (WB 1:5000, Santa Cruz Biotechnology); Alexa Fluor 488 anti-goat/rabbit, Alexa Fluor 594 anti-mouse/rabbit (IF 1:200, Molecular Probes, Eugene, OR, USA).

Western blot analysis. Western blotting was performed as previously described. ${ }^{14}$ Blots were visualized by enhanced chemiluminescence (Amersham Pharmacia Biotech, Oakville, ON, Canada). All western blots were confirmed for equal loading using $0.1 \%(\mathrm{w} / \mathrm{v})$ Ponceau $S$ solution. For quantification purposes, bands of interest were analyzed using the CanoScanLiDE20 image scanner (Canon Canada, Mississauga, ON, Canada).

Immunofluorescence staining. Immunofluorescence staining was performed as follows. Sodium citrate antigen retrieval was performed, followed by treatment with Sudan Black $(0.1 \%$ sudan black in $70 \% \mathrm{EtOH})$ to quench endogenous fluorescence. Sections were preincubated in $5 \%$ horse serum diluted in antibody diluent $(0.04 \%$ sodium azide and $0.008 \%$ gelatin in PBS), to block nonspecific binding, and incubated with primary antibodies overnight at $4{ }^{\circ} \mathrm{C}$. For negative controls, the primary antibody was replaced by a corresponding concentration of mouse or rabbit lgG. Slides were treated with $0.4 \%$ DAPI $\left(4^{\prime}, 6\right.$ diamidino-2-phenylindole) for nuclear detection. Fluorescence images were viewed using $\times 20$ regular and $\times 40$, and $\times 100$ oil immersion objective lens (NA 1.35) and collected using the DeltaVision Deconvolution microscope (Applied Precision, LLC, Issaquah, WA, USA).

Positive and negative cell counts were based on the presence or absence of immunoreactivity of the Ki67 and Mtd antibody. Cell counts were recorded as a percentage of the total cell number in the field where the total cell number was taken as the number of nuclei (DAPI stained) in the trophoblast layer of floating villi or within anchoring columns. For Ki67 and Cyclin E1 quantification in normal and pathological samples, placental sections were stained using a peroxidase-based method as previously described, ${ }^{45}$ and cell counts were recorded as a percentage of either Ki67- or cyclin E1-positive trophoblast cells per field. Five fields of view were analyzed per sample.

TUNEL. The in situ Cell Death Detection kit (Roche Molecular Biochemicals, Indianapolis, IN, USA) was used on the basis of the manufacturer's protocol.

Cell line culture and analysis. Human choriocarcinoma JEG-3 cells were grown in EMEM media (ATCC, Manassas, VA, USA) supplemented with $10 \%$ fetal bovine serum (nonheat inactivated) in $20 \%$ oxygen (standard conditions). 
SNP treatment. JEG-3 cells were seeded into a six-well plate $\left(2 \times 10^{5}\right.$ cells per well) or 96 -well plates ( 5000 cells per well) and treated after $16 \mathrm{~h}$ with SNP (Sigma) (dose range between 1 and $10 \mathrm{mM}$ ).

Trypan blue exclusion assay. Cells were harvested by trypsinization, centrifuged, resuspended in media, and diluted $1: 10$ in $0.4 \%$ Trypan blue solution (Invitrogen, Carlsbad, CA, USA). Blue versus white cells were counted on a hematocytometer. Data are represented as an average of three independent experiments.

Cell viability (MTT). Cell viability was determined by the MTT (Sigma) dyereduction assay. JEG-3 cells seeded into a 96 -well plate ( 5000 cells per well) were subjected to $30 \mu \mathrm{l}$ of MTT dye ( $5 \mathrm{mg} / \mathrm{ml})$ per well, at $0,6,12,24$, and $48 \mathrm{~h}$. The cells were then incubated at $37^{\circ} \mathrm{C}$ for $4 \mathrm{~h}$. The media was subsequently aspirated and $100 \mu$ l of dimethyl sulfoxide (DMSO) were added to each well. Absorbance (relative optical density) was measured at $570 \mathrm{nM}$ using a uQuant microplate spectrophotometer (Bio-Tek Instruments, Winooski, VT, USA). Data are presented as an average of four experiments.

Cell fractionation. JEG-3 cells were grown to $70 \%$ confluence and collected in lysis buffer ( $0.3 \mathrm{M}$ sucrose, $1 \mathrm{M}$ EDTA pH8, $5 \mathrm{mM}$ Mops, $5 \mathrm{mM} \mathrm{KH}_{2} \mathrm{PO}_{4}, 0.1 \% \mathrm{BSA}$ - $\mathrm{pH}$ to 7.4 using $\mathrm{KOH})$ for fractionation. Cells were lysed using a dounce homogenizer, and differentially centrifuged to attain cell fractions: 5 min at $600 \mathrm{~g}$ to precipitate nuclei followed by $12 \mathrm{~min}$ at $10000 \mathrm{~g}$ to pellet light membrane fraction. The resulting supernatant containing the cytoplasmic fraction was lyophilized and all fractions were resuspended in RIPA buffer. Verification of fraction specificity was assessed by western blot detection of specific subcellular markers: $\alpha$-tubulin (cytoplasm), cytochrome $c$ (mitochondria), and lamin A (nuclear membrane). Localization of Mtd to mitochondria. JEG-3 cells $\left(3.0 \times 10^{5}\right)$ were seeded on sterile glass coverslips and allowed to adhere overnight. Cells were then incubated with $100 \mathrm{nM}$ MitoTracker Red CMXRos (M-7512) (Molecular Probes), a dye taken up by active mitochondria, and fixed in $3.7 \%$ formaldehyde. Incubation with primary antibody against Mtd was carried out overnight, and secondary detection was performed as described above for embedded sections.

Construction of stable cell line expressing GFP-hMtdL. Human embryonic kidney (HEK) cell line stably expressing GFP-hMtdL was generated as follows: plasmid-encoding GFP-hMtdL was stably introduced into Flp-In T-Rex-293 cell line (Invitrogen), as described in the manufacturer's protocol. Briefly, the human MtdL gene was amplified from full-length cDNA hMtdL (Open Biosystems) by PCR using the forward primer $5^{\prime}$-ggcgcgccagaggtgctgcggcgctcctcg- $3^{\prime}$ and the reverse primer $5^{\prime}$-cagagagatgacccggatcccg-3'. The PCR was digested by Ascl/BamHI and cloned into pcDNA5/FRT/TO/GFP (a kind gift from Dr. Gingras, SLRI at Mount Sinai Hospital, Toronto). The resulting plasmid was verified by digest and sequencing and finally cotransfected along with pOG44 (Invitrogen) into Flp-In T-Rex-293 cells to induce a site-specific integration event. Hygromycin-resistant clones were obtained under $100-\mu \mathrm{g} / \mathrm{ml}$ hygromycin selection. MtdL was induced in the stable transfected cell line with $0.05-2.5 \mu \mathrm{g} / \mathrm{ml}$ of doxycycline for $36 \mathrm{~h}$.

BrdU incorporation. 5-Bromo-2'-deoxy-uridine labeling was performed in accordance with the manufacturer's protocol (Roche Applied Sciences, made in: Mannheim, Germany; distributed in Indianapolis, IN, USA). Cells were subjected to BrdU for either 20 or $45 \mathrm{~min}$ following either doxycycline $(0.25 \mu \mathrm{g} / \mathrm{ml})$ or MtdL siRNA $(30 \mu \mathrm{M})$ treatments.

Statistical analysis. Statistical analyses were performed using GraphPad Prism 4 software (San Diego, CA). For comparison of data between multiple groups, we used the Kruskal-Wallis test. For comparison between the two groups, we used the Mann-Whitney U-test. Significance was defined as $P<0.05$. Results are expressed as the mean \pm S.E.M. or box and whisker plots showing the median and interquartile ranges.

Acknowledgements. We thank Dr. Dragica Curovic for placental collection and Yuan Wu for her technical assistance. Our appreciation goes out to Dr. Alan Bocking for his constant support. We thank Martin Post for a critical reading of the manuscript. This work was supported by the Canadian Institutes of Health Research (CIHR) Grant (MOP 62865) to Isabella Caniggia. Jocelyn Ray is supported by the CIHR DRA scholarship and the Genesis Foundation's Ontario Graduate Scholarship Program at the University of Toronto. Andrea Jurisicova is the recipient of the CIHR New Investigator award and Isabella Caniggia is the recipient of the Ontario Women's Health Career Award.

\section{Conflict of interest}

The authors declare no conflict of interest.

1. Lea RG, Riley SC, Antipatis C, Hannah L, Ashworth CJ, Clark DA et al. Cytokines and the regulation of apoptosis in reproductive tissues: a review. Am J Reprod Immunol 1999; 42: 100-109.

2. Levy R, Nelson DM. To be, or not to be, that is the question. Apoptosis in human trophoblast. Placenta 2000; 21: 1-13.

3. Smith SC, Baker PN, Symonds EM. Placental apoptosis in normal human pregnancy. Am J Obstet Gynecol 1997; 177: 57-65.

4. Burton GJ, Jauniaux E, Watson AL. Maternal arterial connections to the placental intervillous space during the first trimester of human pregnancy: the Boyd collection revisited. Am J Obstet Gynecol 1999; 181: 718-724.

5. Jaffe R, Jauniaux E, Hustin J. Maternal circulation in the first-trimester human placentamyth or reality? Am J Obstet Gynecol 1997; 176: 695-705.

6. Rodesch F, Simon $P$, Donner $C$, Jauniaux $E$. Oxygen measurements in endometrial and trophoblastic tissues during early pregnancy. Obstet Gynecol 1992; 80: 283-285.

7. Smith SC, Leung TN, To KF, Baker PN. Apoptosis is a rare event in first-trimester placental tissue. Am J Obstet Gynecol 2000; 183: 697-699.

8. Genbacev O, Zhou Y, Ludlow JW, Fisher SJ. Regulation of human placental development by oxygen tension. Science 1997; 277: 1669-1672.

9. Soleymanlou N, Jurisica I, Nevo O, letta F, Zhang X, Zamudio S et al. Molecular evidence of placental hypoxia in preeclampsia. J Clin Endocrinol Metab 2005; 90: 4299-4308.

10. Heazell AE, Crocker IP. Live and let die - regulation of villous trophoblast apoptosis in normal and abnormal pregnancies. Placenta 2008; 29: 772-783.

11. Ray J, Jurisicova A, Caniggia I. IFPA trophoblast research award lecture: the dynamic role of Bcl-2 family members in trophoblast cell fate. Placenta 2008; 30 (Suppl A): S96-S100.

12. Finucane DM, Bossy-Wetzel E, Waterhouse NJ, Cotter TG, Green DR. Bax-induced caspase activation and apoptosis via cytochrome $\mathrm{c}$ release from mitochondria is inhibitable by BCl-XL. J Biol Chem 1999; 274: 2225-2233.

13. Green DR, Reed JC. Mitochondria and apoptosis. Science 1998; 281: 1309-1312.

14. Soleymanlou N, Wu Y, Wang JX, Todros T, letta F, Jurisicova $A$ et al. A novel Mtd splice isoform is responsible for trophoblast cell death in pre-eclampsia. Cell Death Differ 2005; 12: $441-452$.

15. Bonnefoy-Berard N, Aouacheria A, Verschelde C, Quemeneur L, Marcais A, Marvel J. Control of proliferation by Bcl-2 family members. Biochim Biophys Acta 2004; 1644 159-168.

16. Maddika S, Ande SR, Panigrahi S, Paranjothy T, Weglarczyk K, Zuse A et al. Cell survival, cell death and cell cycle pathways are interconnected: implications for cancer therapy. Drug Resist Updat 2007; 10: 13-29.

17. Zinkel S, Gross A, Yang E. BCL2 family in DNA damage and cell cycle control. Cell Death Differ 2006; 13: 1351-1359.

18. Brady HJ, Gil-Gomez G, Kirberg J, Berns AJ. Bax alpha perturbs T cell development and affects cell cycle entry of T cells. EMBO J 1996; 15: 6991-7001.

19. Fujise K, Zhang D, Liu J, Yeh ET. Regulation of apoptosis and cell cycle progression by MCL1. Differential role of proliferating cell nuclear antigen. J Biol Chem 2000; 275 : 39458-39465.

20. Jamil S, Sobouti R, Hojabrpour P, Raj M, Kast J, Duronio V. A proteolytic fragment of Mcl-1 exhibits nuclear localization and regulates cell growth by interaction with Cdk1. Biochem J 2005; 387 (Part 3): 659-667.

21. Knudson CM, Johnson GM, Lin Y, Korsmeyer SJ. Bax accelerates tumorigenesis in p53deficient mice. Cancer Res 2001; 61: 659-665.

22. Hsu SY, Kaipia A, McGee E, Lomeli M, Hsueh AJ. Bok is a pro-apoptotic Bcl-2 protein with restricted expression in reproductive tissues and heterodimerizes with selective antiapoptotic Bcl-2 family members. Proc Natl Acad Sci USA 1997; 94: 12401-12406.

23. Hsu SY, Hsueh AJ. Tissue-specific Bcl-2 protein partners in apoptosis: an ovarian paradigm. Physiol Rev 2000; 80: 593-614.

24. Rodriguez JM, Glozak MA, Ma Y, Cress WD. Bok, Bcl-2-related ovarian killer, is cell cycle-regulated and sensitizes to stress-induced apoptosis. J Biol Chem 2006; 281: 22729-22735.

25. ACOG practice bulletin. Diagnosis and management of preeclampsia and eclampsia Number 33, January 2002. American college of obstetricians and gynecologists. Int $J$ Gynaecol Obstet 2002; 77: 67-75.

26. Roberts JM, Cooper DW. Pathogenesis and genetics of pre-eclampsia. Lancet 2001; 357: 53-56.

27. Gerretsen G, Huisjes HJ, Elema JD. Morphological changes of the spiral arteries in the placental bed in relation to pre-eclampsia and fetal growth retardation. $\mathrm{Br} \mathrm{J}$ Obstet Gynaecol 1981; 88: 876-881.

28. Hung TH, Skepper JN, Charnock-Jones DS, Burton GJ. Hypoxia-reoxygenation: a potent inducer of apoptotic changes in the human placenta and possible etiological factor in preeclampsia. Circ Res 2002; 90: 1274-1281.

29. Soleymanlou N, Jurisicova A, Wu Y, Chijiiwa M, Ray JE, Detmar J et al. Hypoxic switch in mitochondrial myeloid cell leukemia factor-1/Mtd apoptotic rheostat 
contributes to human trophoblast cell death in Preeclampsia. Am J Pathol 2007; 171 496-506.

30. Endl E, Gerdes J. The Ki-67 protein: fascinating forms and an unknown function. Exp Cell Res 2000; 257: 231-237.

31. Lew DJ, Dulic V, Reed SI. Isolation of three novel human cyclins by rescue of G1 cyclin (CIn) function in yeast. Cell 1991; 66: 1197-1206.

32. Sherr CJ, Roberts JM. Living with or without cyclins and cyclin-dependent kinases. Genes Dev 2004; 18: 2699-2711.

33. Brown LM, Lacey HA, Baker PN, Crocker IP. E-cadherin in the assessment of aberrant placental cytotrophoblast turnover in pregnancies complicated by pre-eclampsia. Histochem Cell Biol 2005; 124: 499-506.

34. De Falco M, De Luca L, Acanfora F, Cavallotti I, Cottone G, Laforgia V et al. Alteration of the $\mathrm{Bcl}-2$ : Bax ratio in the placenta as pregnancy proceeds. Histochem $J$ 2001; 33: 421-425.

35. Gao S, Fu W, Durrenberger M, De Geyter C, Zhang H. Membrane translocation and oligomerization of hBok are triggered in response to apoptotic stimuli and Bnip3. Cell Mol Life Sci 2005; 62: 1015-1024.

36. Inohara N, Ekhterae D, Garcia I, Carrio R, Merino J, Merry A et al. Mtd, a novel Bcl-2 family member activates apoptosis in the absence of heterodimerization with $\mathrm{Bcl}-2$ and Bcl-XL. J Biol Chem 1998; 273: 8705-8710.

37. Antonsson B, Montessuit S, Lauper S, Eskes R, Martinou JC. Bax oligomerization is required for channel-forming activity in liposomes and to trigger cytochrome $c$ release from mitochondria. Biochem J 2000; 345 (Part 2): 271-278.
38. Antonsson B, Montessuit S, Sanchez B, Martinou JC. Bax is present as a high molecular weight oligomer/complex in the mitochondrial membrane of apoptotic cells. J Biol Chem 2001; 276: 11615-11623.

39. Dejean LM, Martinez-Caballero S, Guo L, Hughes C, Teijido O, Ducret T et al. Oligomeric $B a x$ is a component of the putative cytochrome $c$ release channel MAC, mitochondrial apoptosis-induced channel. Mol Biol Cell 2005; 16: 2424-2432.

40. Wolter KG, Hsu YT, Smith CL, Nechushtan A, Xi XG, Youle RJ. Movement of Bax from the cytosol to mitochondria during apoptosis. J Cell Biol 1997; 139: 1281-1292.

41. Bartholomeusz G, Wu Y, Ali SM, Xia W, Kwong KY, Hortobagyi G et al. Nuclear translocation of the pro-apoptotic Bcl-2 family member Bok induces apoptosis. $\mathrm{Mol}$ Carcinog 2006; 45: 73-83.

42. Abuhamad AZ. ACOG Practice Bulletin, clinical management guidelines for obstetriciangynecologists. number 98 , October 2008 (replaces Practice Bulletin number 58 , December 2004). Ultrasonography in pregnancy. Obstet Gynecol 2008; 112: 951-961.

43. Hansen AR, Collins MH, Genest D, Heller D, Shen-Schwarz S, Banagon P et al. Very low birthweight placenta: clustering of morphologic characteristics. Pediatr Dev Pathol 2000; 3 : 431-438

44. Redline RW. Placental pathology: a systematic approach with clinical correlations. Placenta 2008; 29 (Suppl A): S86-S91.

45. Caniggia I, Mostachfi H, Winter J, Gassmann M, Lye SJ, Kuliszewski M et al. Hypoxiainducible factor- 1 mediates the biological effects of oxygen on human trophoblast differentiation through TGFbeta(3). J Clin Invest 2000; 105: 577-587. 